\title{
DESPRENDIMIENTO SEROSO DE COROIDES Y DESPRENDIMIENTO DE RETINA POSTERIOR CAPSULOTOMÍA CON YAG-LÁSER
}

\section{CHOROIDAL EFFUSION AND RETINAL DETACHMENT AFTER CAPSULOTOMY WITH YAG-LASER}

\author{
CÁMARA-CASTILLO HG ${ }^{1}$, NAVARRO-LÓPEZ P² ${ }^{2}$ RIVERA-SEMPÉRTEGUI J ${ }^{3}$
}

\begin{abstract}
RESUMEN
Caso Clínico: Mujer de 74 años, que acudió por visión borrosa. Pseudofaca con lente intraocular de cámara anterior en ambos ojos, y opacidad de cápsula posterior de ojo izquierdo, se realizó capsulotomía con YAG-LASER. A los trece días presenta desprendimiento coroideo y desprendimiento de retina que requirió intervención quirúrgica.

Discusión: El indicador para realizar una capsulotomía más usado es la disminución de la agudeza visual sin que exista una indicación precisa de cuando realizarla. Sin embargo; se deben de tener en cuenta las posibles complicaciones como son: desprendimiento de retina regmatógeno, dislocación del lente, uveítis recurrente, hipertensión ocular y complicaciones devastadoras como es el desprendimiento seroso de coroides y desprendimiento de retina.
\end{abstract}

Palabras claves: Capsulotomía, desprendimiento de seroso de coroides, desprendimiento de retina.

\begin{abstract}
Case report: A 74-year-old woman presented complaining of blurred vision in her left eye. She had an anterior chamber lens in both eyes and an opaque posterior capsule in her left eye, for which a YAGLASER capsulotomy was performed. Thirteen days later she re-presented with a choroidal effusion and a retinal detachment requiring surgery.

Discussion: There is no consensus as to the exact time at which a capsulotomy should be done. Timing of the procedure requires evaluation as a whole and consideration of the potential complications of IOL dislocation, recurrent uveitis, ocular hypertension and the most devastating choroidal effusion and retinal detachment (Arch Soc Esp Oftalmol 2006; 81: 333-336).
\end{abstract}

Key words: Capsulotomy, choroidal effusion, retinal detachment.

\footnotetext{
Recibido: 18/3/05. Aceptado: 12/6/06

Servicio de Enfermedades Inflamatorias Oculares y Retina. Asociación para Evitar la Ceguera en México. Hospital Dr. Luis Sánchez Bulnes. México, D.F. México.

1 Licenciado en Medicina.

2 Licenciado en Medicina. Subespecialista en Uveítis.

3 Licenciado en Medicina. Subespecialista en Retina.

En el presente trabajo no existe interés comercial ni aporte financiero alguno.

Correspondencia:

Héctor Cámara Castillo

Vicente García Torres No. 46 San Lucas Coyoacán

México, D.F.

México

E-mail: heccam@gotmail.com / patriv2802@hotmail.com
} 


\section{INTRODUCCIÓN}

El Yttrium Aluminium Garnet (YAG) laser se utiliza para la apertura de la cápsula posterior en pacientes que han sido sometidos a cirugía de catarata. La disminución de la agudeza visual es el parámetro más preciso para realizar una capsulotomía (1) y se han descrito múltiples complicaciones. El propósito de este trabajo es describir el caso de un paciente con desprendimiento seroso de coroides y desprendimiento de retina posterior a capsulotomía, y discutir los riesgos que conlleva dicho procedimiento, así como su manejo y pronóstico visual.

\section{CASO CLÍNICO}

Mujer de 74 años de edad diabética tipo II de 15 años de evolución, tratada con hipoglucemiantes orales, que acude por disminución de la agudeza visual. Se realizó cirugía extracapsular de catarata en 1992 en ojo derecho (OD), y en el 2001 en ojo izquierdo (OI) con lente de cámara anterior de polimetilmetacrilato y cápsula íntegra sin conocerse la causa para no usar un LIO de cámara posterior.

Exploración funcional presenta una agudeza visual (AV) de 0,6 en OD y de 0,2 en OI, presión ocular por aplanación (PIO) de 14 y $15 \mathrm{~mm} \mathrm{Hg}$, respectivamente. En la biomicroscopía en el OD presenta lente intraocular (LIO) en la cámara anterior (CA) sin más alteraciones. En el polo anterior del OI se aprecia una LIO en la CA, con una opacidad de cápsula posterior de ++ , resto sin alteraciones.

Se realizó capsulotomía con YAG-LASER en el OI con poder de 5,2 mJ (31 disparos). Se creó una capsulotomía circular de $2 \times 2 \mathrm{~mm}$. Tres días después del tratamiento presentaba una AV de 0,7 en el OD y 0,5 en el OI, polo anterior y polo posterior sin alteraciones.

Trece días tras la capsulotomía, refirió la disminución súbita de la agudeza visual del OI. A la exploración presentaba una AV de movimiento de manos (MM) y PIO de $3 \mathrm{mmHg}$, una capsulotomía circular, polo anterior con signos de inflamación, con pigmento en vítreo anterior proyectándose hacia la cara posterior del LIO. Polo posterior con desprendimiento coroideo (DC) con bolsas superiores nasal y temporal, sin toque entre ellas, desprendimiento regmatógeno de retina de meridiano de las IX a las II con afectación macular parcial, encon- trándose la lesión causal (desgarro en herradura) en el meridiano de las XII (figs. 1 y 2). Documentado por ecografía con una longitud axial ocular de 23,12 mm (fig. 3).

Se inició el tratamiento del desprendimiento coroideo con prednisona $1,5 \mathrm{mg} / \mathrm{kg} /$ día vía oral y se inyectó en espacio subtenoniano $2,71 \mathrm{mg}$ de acetato de betametasona y $3,0 \mathrm{mg}$ de fosfato sódico de betametasona (Celestote soluspan, Schering Plough, Mexico, D.F.). A los catorce días, se observó una PIO de $8 \mathrm{mmHg}$ y una leve disminución en el tamaño del DC, por lo que se aplicó una segunda dosis de $2,71 \mathrm{mg}$ de acetato de betametasona y 3,0

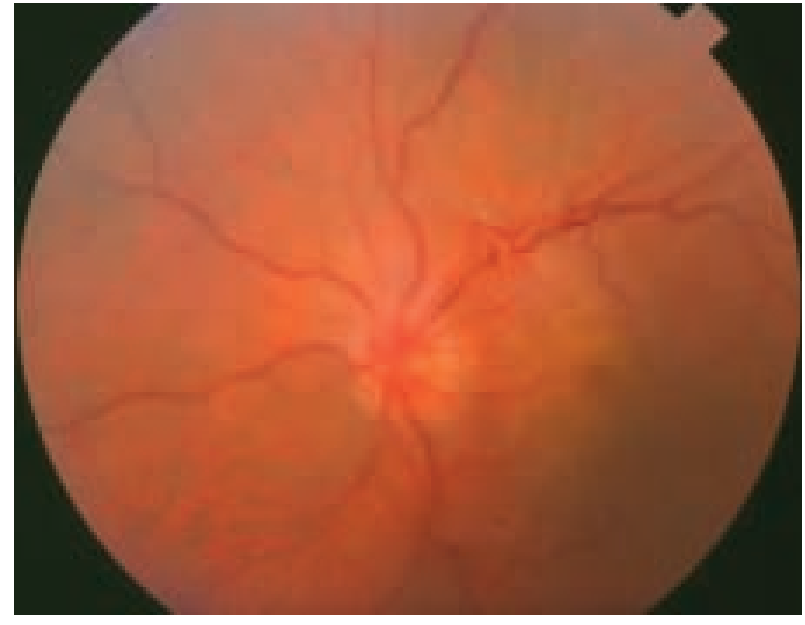

Fig. 1: DR regmatógeno. Polo posterior de ojo izquierdo en el que se observa tortuosidad vascular con elevación generalizada del polo posterior.

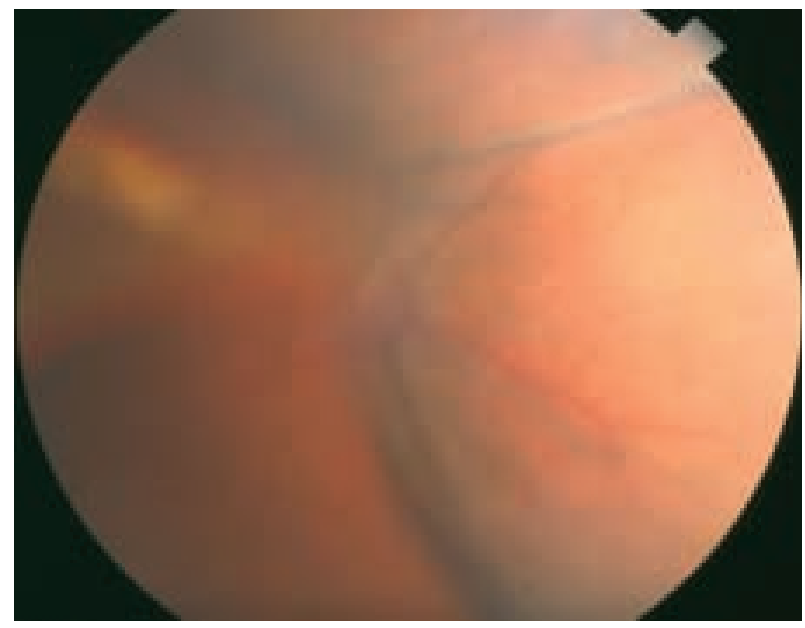

Fig. 2: DC. Región temporal de ojo izquierdo, en el que se observan bolsas temporales lisas de aspecto naranja. 


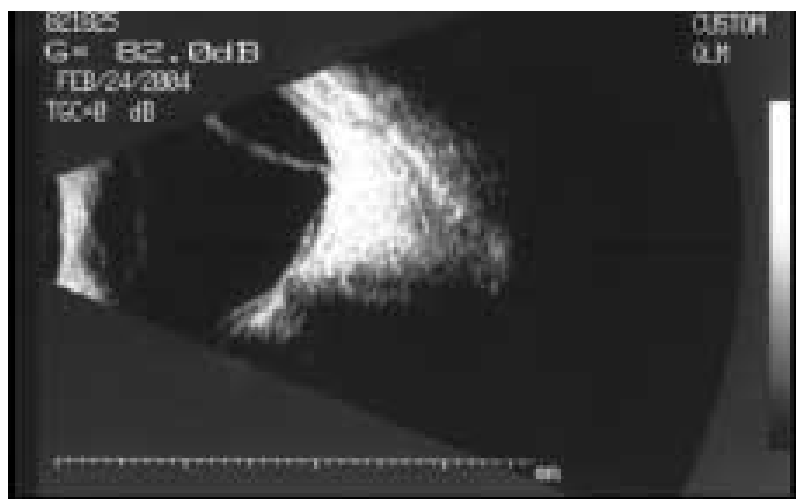

Fig. 3: Ecografía modo B. Se observa desprendimiento coroideo abolsado y DR en la mácula.

mg de fosfato sódico de betametasona subtenoniano (fig. 4).

A los diecinueve días, presentó una menor inflamación en el desprendimiento coroideo no resuelto con tratamiento convencional, además del desprendimiento de retina, por lo que se decidió realizar una intervención quirúrgica mediante el drenaje del desprendimiento coroideo, vitrectomía y aceite de silicona en ojo izquierdo.

Transcurridos tres meses tras la cirugía en el OI presentaba una AV de CD $50 \mathrm{~cm}$, PIO de $9 \mathrm{mmHg}$, midriasis media, LIO en polo anterior, retina aplicada con áreas de fibrosis sin tracción y silicona transparente en cavidad vítrea (fig. 5).

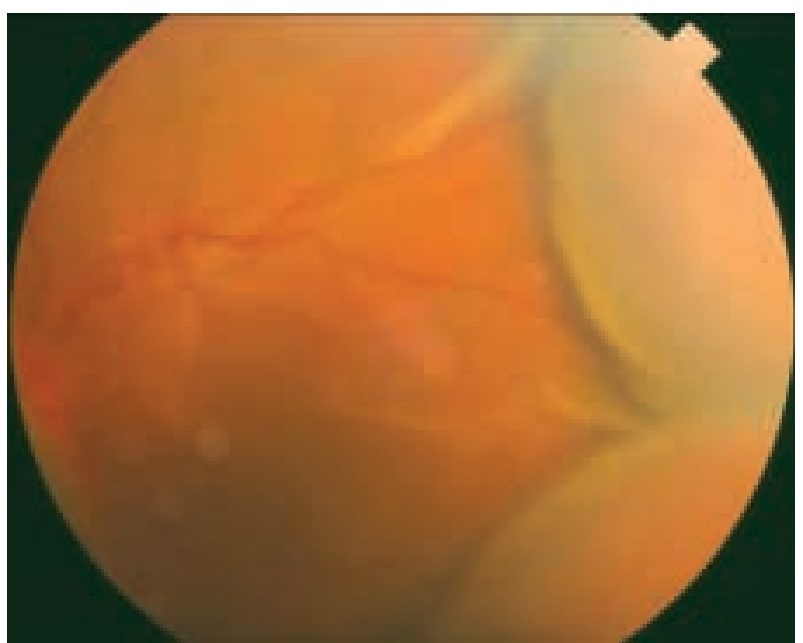

Fig. 4: DC. Región temporal de ojo izquierdo, persistían las bolsas coroideas.

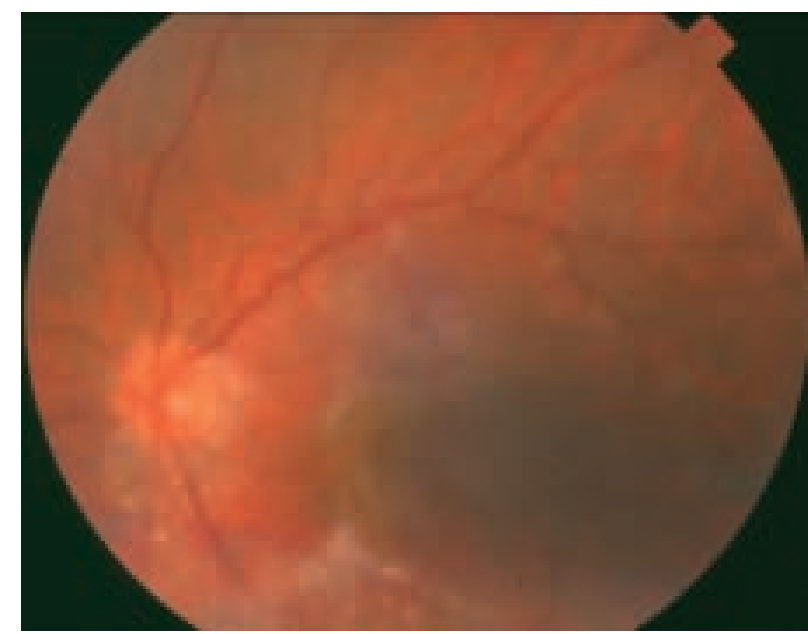

Fig. 5: Posquirúrgico. Región temporal con retina aplicada y bolsas coroideas ya drenadas.

\section{DISCUSIÓN}

La decisión para la realización de una capsulotomía está sujeta a diferentes criterios, algunos autores sugieren la capacidad visual como el parámetro más fiable para realizarla (1). Sin embargo hasta el momento no existe una indicación precisa de cuando realizar una capsulotomía.

Se han publicado complicaciones importantes después de este procedimiento como son: dislocación del lente a cavidad vítrea hasta 16 meses posteriores al procedimiento (2), escleritis y uveítis recurrente asociada, aparentemente relacionado al desplazamiento de las hápticas del lente con inflamación del cuerpo ciliar, y que respondió a tratamiento anti-inflamatorio (3).

Múltiples trabajos han sido publicados en donde se establece una relación causal entre capsulotomía con YAG-LASER y desprendimiento regmatógeno de retina, encontrándose un riesgo de $5,8 \%$ a 5 años; sin embargo, se ha constatado un menor porcentaje ( $1,2 \%$ a 5 años) en los pacientes que previamente a la cirugía fueron sometidos a revisión de la periferia y tratamiento de lesiones predisponentes (4).

Existe la publicación de un DC aislado asociado a una capsulotomía con YAG-LASER, en un paciente de 79 años, sin ningún factor predisponente, que se presentó 5 días posteriores al procedimiento y que se resolvió 15 días después sin tratamiento (5). 
La capsulotomía en un paciente con una LIO de CA es un caso poco común, ya que ésta se implanta en caso de no existir un buen remanente capsular o la ausencia total de la cápsula posterior; se desconoce el motivo por el cual se colocó LIO de CA teniendo la cápsula posterior, ya que no se realizó el procedimiento en nuestra institución. Algunas posibles explicaciones a esto podrían ser: inestabilidad zonular, ruptura de cápsula posterior en sector inferior y no contar con LIO de cámara posterior.

La LIO de CA, aunado al número, potencia y tamaño de la capsulotomía probablemente tengan una relación causal con el DR regmatógeno y el DC que se presentó 13 días después del procedimiento y en el cuál no se encontró otra patología coexistente que podría surgir como causante de estas complicaciones.

Se inició tratamiento con esteroides, y al controlarse el cuadro inflamatorio agudo, aun sin respuesta el tamaño y localización del DC se decidió realizar la intervención quirúrgica. Creemos que la presencia de un LIO de CA con cápsula posterior íntegra, situación poco común; y la necesidad de capsulotomía con un desplazamiento de vítreo anterior pudo relacionarse con el desprendimiento de retina y con la hipotensión precipitarse el DC, sin embar- go existen reportes previos de DC sin DR-regmatógeno, por lo que este no explica del todo el DC.

Por ello en el momento de realizar una capsulotomía es importante tener en cuenta los riesgos a los que se somete el paciente, y la posibilidad de requerir una intervención quirúrgica con un pobre pronóstico visual.

\section{BIBLIOGRAFÍA}

1. Hayashi K, Hayashi H, Nakao F, Hayashi F. Correlation between posterior capsule opacification and visual function before and after Neodymium: YAG laser posterior capsulotomy. Am J Ophthalmol 2003; 136: 720-726.

2. Petersen AM, Bluth LL, Campion M. Delayed posterior dislocation of silicone plate-haptic lenses after neodymium: yag capsulotomy. J Cataract Refract Surg 2000; 26: 1827-1829.

3. Herbort CP. Uveoscleritis after excessive neodymium:YAG laser posterior capsulotomy. J Cataract Refract Surg 1994; 20: 80-81.

4. Ranta P, Tommila P, Kivela T. Retinal breaks and detachment alter neodymium: YAG laser posterior capsulotomy: five-year incidence in a prospective cohort. J Cataract Refract Surg 2004; 30: 58-66.

5. Auw-Haedrich C, Wiek J. Choroidal effusion after YAG laser capsulotomy. Eye 2001; 15: 794-795. 